\title{
Analysis of demographic and clinical characteristics of primary myelofibrosis and post-polycythemia vera/essential thrombocythemia myelofibrosis patients
}

\author{
(1) Pınar Akyol, (1) Abdulkerim Yıldız, (1) Murat Albayrak, (1) Hacer Berna Afacan Öztürk, (1) Senem Maral, \\ (1) Merih Reis Aras, (1) Fatma Yılmaz, (1) Buğra Sağlam, (1) Mesut Tığlığlu, (1) Ümit Yavuz Malkan
}

University of Health Sciences Turkey, Diskapi Yildirim Beyazit Training and Research Hospital, Clinic of Hematology, Ankara, Turkey

Date submitted:

03.07.2020

Date accepted:

27.10.2020

Online publication date:

15.06.2021

\section{Corresponding Author:}

Pınar Akyol, M.D., University of Health Sciences Turkey, Diskapi Yildirim

Beyazit Training and Research Hospital, Clinic of Hematology, Ankara, Turkey

ORCID:

orcid.org/0000-0003-3829-289X

Keywords: Myeloproliferative disorders, primary myelofibrosis, polycythemia vera

\begin{abstract}
Aims: Primary myelofibrosis (PMF) and post-polycythemia vera (PV) or essential thrombocythemia (ET) myelofibrosis (MF) have many common features in terms of clinical course and laboratory findings. However, there are insufficient studies showing the etiological or morphological differences between these patients. The aim of this study was to compare the hemogram parameters, routine biochemical markers, presence of Janus kinase-2 (JAK2) V617F gene mutation and allele burden, and spleen sizes and also to analyze the clinical courses of these two patient groups regarding to thromboembolic complications, leukemic transformation, overall survival (OS), and treatment modalities.
\end{abstract}

Methods: This retrospective study included patients who were diagnosed with PMF and post-PV/ET MF in the Diskapi Yildirim Beyazit Training and Research Hospital between 2008 and 2019. PMF and post-PV/ET MF patients were compared in terms of demographic data, laboratory parameters and clinical features.

Results: Evaluation was made on a total of 31 patients ( $16 \mathrm{PMF}, 15$ post-PV/ET MF). The mean follow-up period was 31.1 months. JAK-2 mutation was detected in $62.5 \%$ of PMF patients and in $80 \%$ of post-PV/ET MF patients. Thromboembolic complications developed in $12.5 \%$ of PMF patients and in $13.3 \%$ of post-PV/ET MF patients. The mean OS of patients was 28.7 months in the PMF group and 18.5 months in the post-PV/ET MF group. No significant difference was observed between the two groups in terms of OS.

Conclusions: We found that clinical characteristics of PMF and post-PV/ET MF patients such as hemogram and biochemical values, absence of JAK-2 mutation and allele burden, and spleen sizes were similar. We also found that patients showed a similar clinical course in terms of thromboembolic complications, acute leukemia transformation and survival.
Introduction

Myelofibrosis (MF), characterized by fibrosis in the bone marrow, could be de novo [primary MF (PMF)], as well as developing in the clinical course of polycythemia vera $(\mathrm{PV})$ or essential thrombocythemia (ET) (1). PMF is included in the category of "myeloproliferative neoplasms" (MPN) in the World Health Organization (WHO) classification of myeloid neoplasms and acute leukemia (2). The other group, which is categorized as post-PV MF and post-ET MF, is diagnosed according to the criteria determined by the International Working Group for MPN Research and Treatment (IWG-MRT) (3).

Patients present with clinically characteristic laboratory features such as anemia, splenomegaly due to extramedullary hematopoiesis, increased serum lactate dehydrogenase (LDH) level and stromal changes in the bone marrow (eg collagen fibrosis, osteosclerosis) (4). The most common laboratory abnormality in PMF is anemia and in $50 \%$ of patients, hemoglobin $(\mathrm{Hb})$ is $<10 \mathrm{~g} / \mathrm{dL}$ at the time of diagnosis. The most common

${ }^{\circledR}$ Copyright 2021 by the University of Health Sciences Turkey, Gülhane Faculty of Medicine / Gülhane Medical Journal published by Galenos Publishing House. 
symptoms are early satiety, fatigue, abdominal distention and left upper quadrant pain associated with splenomegaly. Unlike PMF, these symptoms are observed in PV and ET patients after the development of MF (5).

In PMF, scoring systems such as Dynamic International Prognostic Scoring System (DIPSS) or DIPSS-plus are generally used to determine the prognosis. DIPSS is based on five clinical and laboratory parameters: age $>65$, white blood cell (WBC) count $>25 \times 10^{9} / \mathrm{L}$, peripheral blast cells $>1 \%, \mathrm{Hb}<10 \mathrm{~g} / \mathrm{dL}$ and the presence of B symptoms. In addition to DIPSS, cytogenetic data, platelet count and blood transfusion requirement are included in the DIPSS-plus $(6,7)$. However, these systems are insufficient to determine the prognosis in post-PV/ET MF (8). An alternative prognostic model for these patients is required to determine the prognosis and treatment decision.

PMF is a disease of the elders and the median survival is 4-7 years (9). Transformation to MF usually occurs 7-20 years after diagnosis in ET and PV patients and this leads to decreased survival (10). Causes of death include leukemic transformation, thrombosis, infections, bleeding and complications of portal hypertension (11).

PMF and post-PV/ET MF have many common features with clinical course and laboratory findings such as extramedullary hematopoiesis resulting in bone marrow fibrosis and splenomegaly, varying degrees of cytopenia(s) and a leukoerythroblastic blood picture (10). However, there are insufficient studies showing the etiological or morphological differences between these patients. In this context, the aim of this study was to contribute to the literature by comparing PMF and PV/ET patients who developed MF regarding to hemogram parameters, routine biochemical markers, presence of Janus kinase-2 (JAK-2) V617F gene mutation and allele burden, and spleen sizes. At the same time, to compare the clinical course of these two patient groups, thromboembolic complications, leukemic transformation, overall survival (OS), and treatment modalities were examined.

\section{Methods}

This retrospective study included 31 patients who were diagnosed with PMF and post-PV/ET MF in the Hematology Department of Diskapi Yildirim Beyazit Training and Research Hospital between 2008 and 2019. All patients over the age of 18 years were included in the study.

No exclusion criteria were determined in the study. All data of the living patients were examined until the last outpatient control and all data of the dead patients were examined until the date of death. OS was determined as the time from the date of diagnosis until the date of death.

The diagnosis of PMF was made according to the WHO criteria (2), and the IWG-MRT group criteria were used for the diagnosis of PPV-MF and PET-MF (3). The two groups were compared in terms of demographic and clinical features. The diagnosis date, demographic and clinical features, physical examination findings, mutation analyses, treatment management and follow-up times of all the patients were recorded. Hematological parameters including $\mathrm{Hb}$, hematocrit (Hct), leukocyte (WBC), neutrophil, lymphocyte, monocyte, platelet, platelet distribution width (PDW), mean platelet volume (MPV), LDH, ferritin and vitamin B12 levels were examined. DIPSS (7) was used to determine the prognosis of the patients. Survival analyses were performed.

\section{Statistical Analysis}

Data obtained in the study were statistically analyzed using SPSS Statistics version 20 Software (IBM, Armonk, NY, USA). Descriptive data were given as percentages. The independent sample t-test (t-table value) was used to compare two independent groups with normal distribution of the measurement values, and the Mann-Whitney $U$ test (Z-table value) was applied to data not showing normal distribution. $x^{2}$-cross tables were used to examine the relationships between qualitative variables. The Cox regression model was also applied to the parameters (age, gender, presence of splenomegaly, size of spleen, JAK-2 mutation status and allele burden, bone marrow fibrosis grade, $\mathrm{Hb}$, Hct, leukocyte (WBC), neutrophil, lymphocyte, monocyte, platelet, PDW, MPV, LDH, ferritin and vitamin B12 levels, platelet to lymphocyte ratio, neutrophil to lymphocyte ratio, monocyte to lymphocyte ratio, DIPSS and presence of thromboembolic complication) evaluated by univariate analysis. Two-sided p-values $<0.05$ were considered statistically significant. The Kaplan-Meier test was applied for survival analysis. The logrank (Mantel-Cox) test was used for OS comparisons between the patient groups.

All procedures performed in this study were conducted in accordance with the ethical standards of the institutional and/ or national research committee and the 1964 Declaration of Helsinki and its subsequent amendments or comparable ethical standards. Approval for this study was granted by the Diskapi Yildirim Beyazit Training and Research Hospital Local Ethics Committee (protocol number: 90/06, date: 22.06.2020).

\section{Results}

Evaluation was made on a total of 31 patients, including 16 PMF and 15 post-PV/ ET MF. The demographic and clinical characteristics of the patient groups are given in Table 1 (the data of the post-PV/ET MF group are the data after MF development).

The PMF group comprised of seven (43.7\%) females and nine $(56.2 \%)$ males with a mean age of $66.50 \pm 11.72$ years. The post-PV/ET MF group comprised of seven (46.6\%) females and eight $(53.4 \%)$ males with a mean age of $57.07 \pm 15.96$ years. 
Splenomegaly was detected at the time of diagnosis in all PMF and post-PV/ET MF patients. When the size of the spleen was examined, there was no statistically significant difference between the two groups.

JAK-2 V617F gene mutation was detected in 10 (62.5\%) of PMF patients and 12 (80\%) of post-PV/ET MF patients. In terms of JAK-2 V617F mutation positivity, there was no statistically significant difference between the two groups. JAK-2 V617F mutation allele burden of $\geq 60 \%$ was detected in $70 \%$ of PMF patients and in $90 \%$ of post-PV/ET MF patients. The allele burden was not determined to affect OS in patients with MF.

When the patients were examined in terms of the treatment they received, hydroxyurea $(\mathrm{HU})$ in $13(81.3 \%)$ and ruxolitinib in three $(18.7 \%)$ of PMF patients were preferred as the first-line

\begin{tabular}{|c|c|c|c|}
\hline Variable & PMF $(n=16)$ & Post-PV/ET MF (n=15) & p \\
\hline Age year $($ mean $\pm S D)$ & $66.50 \pm 11.72$ & $57.07 \pm 15.96$ & 0.184 \\
\hline \multicolumn{4}{|l|}{ Gender n (\%) } \\
\hline Female & $7(43.7)$ & $7(46.6)$ & \multirow{2}{*}{0.5} \\
\hline Male & $9(56.2)$ & $8(53.4)$ & \\
\hline \multicolumn{4}{|l|}{ Splenomegaly $n(\%)$} \\
\hline Yes & $16(100)$ & $15(100)$ & \multirow{2}{*}{0.2} \\
\hline No & 0 & 0 & \\
\hline Median spleen size mm (min-max) & $211.07(151-295)$ & $205.9(142-280)$ & 0.738 \\
\hline \multicolumn{4}{|l|}{ JAK-2 V617F mutation n (\%) } \\
\hline Positive & $10(62.5)$ & $12(80)$ & \multirow[b]{2}{*}{0.29} \\
\hline Negative & $6(37.5)$ & $3(20)$ & \\
\hline \multicolumn{4}{|l|}{ JAK-2 mutant allele burden $\mathrm{n}(\%)$} \\
\hline $0-29 \%$ & $1(10)$ & $1(10)$ & \multirow{3}{*}{0.9} \\
\hline $30-59 \%$ & $2(20)$ & $1(10)$ & \\
\hline $60-100 \%$ & $7(70)$ & $9(90)$ & \\
\hline Bone marrow fibrosis grade median (min-max) & $3.0[2.0-4.0]$ & $3.0[2.0-4.0]$ & 0.727 \\
\hline $\mathrm{Hb}$ g/dL (mean $\pm \mathrm{SD})$ & $11.78 \pm 2.77$ & $11.05 \pm 2.47$ & 0.447 \\
\hline Hct (mean \pm SD) & $36.46 \pm 8.98$ & $34.27 \pm 8.74$ & 0.497 \\
\hline WBC count, $\times 10^{9} / L$ median (min-max) & 21.9 [4.8-43.3] & $7.5[3.8-43.2]$ & 0.055 \\
\hline Neutrophil count, $\times 10^{9} / L$ median (min-max) & $17.7[2.5-37.5]$ & $5.2[1.5-34.9]$ & 0.085 \\
\hline Lymphocyte count, $x 10^{9} / \mathrm{L}$ median (min-max) & $2.2[0.6-8.0]$ & $1.6[0.7-3.9]$ & 0.226 \\
\hline PLT count $\times 10^{9} / L$ median (min-max) & $261.5[119.0-1074.0]$ & $293.0[126.0-1166.0]$ & 0.502 \\
\hline PLR median (min-max) & $127.9[18.9-716.0]$ & $244.3[50.0-790.8]$ & 0.323 \\
\hline NLR median (min-max) & $7.5[1.8-23.7]$ & $3.7[0.9-15.8]$ & 0.429 \\
\hline MLR median (min-max) & $0.3[0.1-1.1]$ & $0.3[0.1-0.5]$ & 0.553 \\
\hline LDH U/L median (min-max) & $663.0[212.0-1157.0]$ & $433.0[271.0-1467.0]$ & 0.141 \\
\hline Ferritin ng/mL median (min-max) & $96.6[7.6-602.0]$ & $133.2[8.5-967.0]$ & 0.232 \\
\hline Vitamin B12 pg/mL median (min-max) & $518.46 \pm 208.36$ & $451.57 \pm 269.63$ & 0.480 \\
\hline \multicolumn{4}{|l|}{ DIPSS score $\mathrm{n}(\%)$} \\
\hline Low & $3(18.8)$ & $2(13.3)$ & \multirow{4}{*}{0.2} \\
\hline Intermediate-1 & $5(31.3)$ & $10(66.7)$ & \\
\hline Intermediate-2 & $4(25)$ & $3(20)$ & \\
\hline High & $4(25)$ & $0(0)$ & \\
\hline \multicolumn{4}{|l|}{ Thromboembolic complication $\mathrm{n}(\%)$} \\
\hline Yes & $2(12.5)$ & $2(13.3)$ & \multirow{2}{*}{0.945} \\
\hline No & $14(87.5)$ & $13(86.7)$ & \\
\hline Mean follow-up period (month) & 29.3 & 33.0 & 0.81 \\
\hline \multicolumn{4}{|l|}{ Final situation n (\%) } \\
\hline Alive & $11(68.8)$ & $12(80)$ & \multirow{2}{*}{0.24} \\
\hline Exitus & $5(31.2)$ & $3(20)$ & \\
\hline
\end{tabular}


treatment. The median follow-up period of these patients with the first-line treatment was 13.5 months (range, 1-92 months). During this follow-up period, $31.5 \%$ of the patients responded to the first-line treatment and the treatments are still continuing. In $62.5 \%$ of patients, second-line treatment was initiated due to adverse effects or unresponsiveness. In the second-line treatment, ruxolitinib was preferred in four (80\%) patients and anagrelide was started in one $(20 \%)$ patient. In the treatment of patients in the post-PV/ET MF group, HU treatment was continued in seven (46.7\%) patients after MF development and ruxolitinib treatment was initiated in 8 (53.3\%) patients. In this group, the median duration of first-line treatment was 16.5 months (range, 2-82 months). During this period, the response rate to the treatment was $66.6 \%$ and the treatments are still ongoing. In $13.3 \%$ of patients, the second-line treatment was initiated due to adverse effects or unresponsiveness. As a second-line treatment, one (33.3\%) patient underwent allogeneic stem cell transplantation (ASCT), interferon-alpha was initiated in one (33.3\%) patient and ruxolitinib was preferred in one $(33.3 \%)$ patient. The detailed statistics of the patient treatments are given in Table 2.

Throughout the follow-up period, thromboembolic

\begin{tabular}{|c|c|c|}
\hline Variable & $\begin{array}{l}\text { PMF } \\
(n=16)\end{array}$ & $\begin{array}{l}\text { Post-PV/ET } \\
\text { MF }(n=15)\end{array}$ \\
\hline \multicolumn{3}{|l|}{ First-line treatment $\mathrm{n}(\%)$} \\
\hline Hydroxyurea & $13(81.3)$ & $7(46.7)$ \\
\hline Ruxolitinib & $3(18.7)$ & $8(53.3)$ \\
\hline $\begin{array}{l}\text { First-line treatment duration } \\
\text { day, median (min-max) }\end{array}$ & $\begin{array}{l}13.5 \\
{[1.0-92.0]}\end{array}$ & $\begin{array}{l}16.5 \\
{[2.0-82.0]}\end{array}$ \\
\hline \multicolumn{3}{|l|}{$\begin{array}{l}\text { Hydroxyurea (as } 1^{\text {st }} \text { line } \\
\text { treatment) } n(\%)\end{array}$} \\
\hline Continues & $3(23)$ & $5(71.4)$ \\
\hline $\begin{array}{l}\text { Unresponsiveness/inadequate } \\
\text { response }\end{array}$ & $3(23)$ & $1(14.2)$ \\
\hline Side effect & $3(23)$ & \\
\hline Exitus & $4(31)$ & $1(14.2)$ \\
\hline \multicolumn{3}{|l|}{$\begin{array}{l}\text { Ruxolitinib (as } 1^{\text {st }} \text { line } \\
\text { treatment) } n(\%)\end{array}$} \\
\hline Continues & $2(66.6)$ & $5(62.5)$ \\
\hline $\begin{array}{l}\text { Unresponsiveness/inadequate } \\
\text { response }\end{array}$ & - & $1(12.5)$ \\
\hline Side effect & - & - \\
\hline Exitus & $1(33.3)$ & $2(25)$ \\
\hline \multicolumn{3}{|l|}{ Second-line treatment $n(\%)$} \\
\hline ASCT & - & $1(33.3)$ \\
\hline Anagrelide & $1(20)$ & - \\
\hline INF-alfa & - & $1(33.3)$ \\
\hline Ruxolitinib & $4(80)$ & $1(33.3)$ \\
\hline \multicolumn{3}{|c|}{$\begin{array}{l}\text { ASCT: Allogenic stem cell transplantation, INF: Interferon, PV/ET: } \\
\text { Polycythemia vera/essential thrombocythemia, MF: Myelofibrosis, PMF: } \\
\text { Primary myelofibrosis, min-max: Minimum-maximum }\end{array}$} \\
\hline
\end{tabular}

complications developed in $12.5 \%$ of PMF patients and in $13.3 \%$ of post-PV/ET MF patients. There was no statistically significant difference between the two groups in terms of thromboembolic complications. Acute myeloid leukemia transformation was observed in one $(6.25 \%)$ patient from the PMF group during the follow-up period.

The mean follow-up was 31.1 months (range, 1-107.5 months). The mean OS of patients was 28.7 months (range, 1-93.4 months) in the PMF group, and 18.5 months (range, 1.2-107.5 months) in the post-PV/ET MF group. As a result of the log-rank (Mantel-Cox) test, no significant difference was observed between the two groups in terms of OS $(p>0.05)$. During the follow-up period, mortality developed in $31.2 \%$ of the PMF patients and in $20 \%$ of the post-PV/ET MF patients.

\section{Discussion}

MF is characterized by fibrosis in the bone marrow stroma and extramedullary hematopoiesis. Philadelphia chromosome negative MPNs all tend to progress to the myelofibrotic stage. Post-PV MF occurs in about $20 \%$ of PV patients at least 10 years after the diagnosis. In contrast, post-ET MF is less common, occurring in $<1 \%$ of cases in 10 years and $<10 \%$ in 15 years of follow-up (1). In contrast to the data in literature, the frequency of MF transformation was observed to be higher in the ET patients. This difference may be due to the fact that some patients were mistakenly diagnosed with ET while they were pre-fibrotic MF in the past. In the diagnostic criteria revised in 2016, WHO recommended performing bone marrow biopsy for the diagnosis of ET to prevent this situation and bone marrow findings were accepted as the major criteria.

Approximately $65 \%$ of PMF patients have JAK-2 V617F gene mutation. In a previous study, JAK-2 V617F mutation positivity was determined in all post-PV MF patients and in $60 \%$ of PMF patients (1). In another study, the frequency of JAK-2 V617F mutation, similar to the previous study, was detected in both PMF and post-PV MF patients (12). In the current study, JAK-2 V617F mutation was detected in $62.5 \%$ of the PMF patients and in $80 \%$ of the post-PV/ET MF patients, which was consistent with the literature.

Almost all of the current study patients with both PMF and post-PV/ET MF had an allele burden of $\geq 60 \%$. However, no effect of allele burden on OS was detected in this study. Similarly, in a study conducted in the Mayo Clinic, no correlation with the mutant allele burden and increased risk of leukemic transformation or splenectomy was observed in PMF patients (13).

Mutations in calreticulin (CALR) (20-25\% of patients) and MPL (myeloproliferative leukemia virus oncogene) $(5-10 \%$ of patients) genes have also been observed in PMF. Apart from these, spot mutations have been detected in myeloid genes such as ASXL1, TET2, EZH2, IDH1/IDH2 in $80 \%$ of patients 
(14). These genetic variations seen in PMF are considered to be the reason for the heterogeneity of the disease in terms of clinical course and OS. For example, CALR-mutant PMF patients have a more indolent clinical course (15). In contrast, high risk of leukemic transformation has been detected in PMF patients without JAK-2, CALR or MPL mutation (16). As CALR and MPL mutation analysis was not available in our hospital until 2018, these cytogenetic tests could not be applied to all the current study patients, so they were not included in the statistical studies.

OS in PMF is worse than in other classic MPNs, ranging from 3.5 to five years (17).

When MF develops during the course of PV or ET, the patient's quality of life deteriorates due to increased constitutional symptoms and their survival is shortened due to extramedullary hematopoiesis and cytopenia-related reasons (9). Although most share clinical features, post-PV/ET MF patients may have significantly different disease characteristics in terms of presentation or prognosis than patients with PMF. For example, post-PV/ET MF is a slow-progressing clonal transformation from a proliferative neoplasm to bone marrow failure, while PMF has a faster and more aggressive clinical course (1). The expected life expectancy of PV and ET, which generally shows a benign clinical course, is almost the same as that of the general population, but the development of MF shortens survival. In the current study, no statistically significant difference was observed. Similarly, in a study by Boiocchi et al. (1) on PMF and post-PV MF patients, there was no difference between the two groups in terms of survival.

Acute leukemia transformation is most frequently observed in PMF among the MPNs. The incidence of acute leukemia transformation in the first 10 years after diagnosis varies between $10 \%$ and $20 \%(18,19)$. The leukemia transformation risk of post-PV/ET MF patients is also similar to that of PMF patients (17). In one of the largest PMF studies, acute leukemia transformation was observed at a rate of $3.9 \%$ in a total of 2,333 PMF patients (17). Leukemia transformation was observed in only one $(6.25 \%)$ of the 16 patients with PMF in the current study, and in the post-PV/ET MF patients, no leukemia transformation was observed during follow-up.

Although ASCT is the only curative treatment for PMF or post-PV/ET MF, palliative treatment options such as $\mathrm{HU}$ are more frequently preferred (20). Treatment of MF patients is focused on reducing constitutional symptoms associated with splenomegaly. In this context, the JAK $1 / 2$ inhibitor, ruxolitinib, is successfully used (21). While the most preferred agent in the first-line treatment in the current study PMF patients was $\mathrm{HU}$, ruxolitinib was started in approximately half of the PV and ET patients who developed MF.

The retrospective design of the study was the most important limitation of this study. Other disadvantages were the relatively short follow-up period and the low number of patients. The number of cases was too low for the comprehensive comparisons that were aimed. Cytogenetic studies have also gained importance in the prognosis of these diseases. The impact of CALR and MPL mutations on fibrosis is controversial and more studies are needed to clarify this relationship.

\section{Conclusion}

We found that clinical characteristics of PMF and post-PV/ ET MF patients such as hemogram and biochemical values, absence of JAK-2 V617F mutation and its allele burden, and spleen sizes were similar. We also found that patients showed a similar clinical course in terms of thromboembolic complications, acute leukemia transformation and survival.

Moreover, it should be kept in mind that if MF develops in PV and ET patients who have good clinical course, prognosis worsens and survival decreases. Therefore, these patients should be followed in terms of MF.

\section{Ethics}

Ethics Committee Approval: Approval for this study was granted by the Diskapi Yildirim Beyazit Training and Research Hospital Local Ethics Committee (protocol number: 90/06, date: 22.06.2020).

Informed Consent: Retrospective study.

Peer-review: Externally peer-reviewed.

\section{Authorship Contributions}

Surgical and Medical Practices: M.A., Concept: P.A., M.A., Design: A.Y., Data Collection or Processing: S.M., M.R.A., Analysis or Interpretation: H.B.A.Ö., F.Y., Ü.Y.M., Literature Search: B.S., M.T., Writing: P.A.

Conflict of Interest: No conflict of interest was declared by the authors.

Financial Disclosure: The authors declared that this study received no financial support.

\section{References}

1. Boiocchi L, Mathew S, Gianelli U, et al. Morphologic and cytogenetic differences between post-polycythemic myelofibrosis and primary myelofibrosis in fibrotic stage. Mod Pathol. 2013;26:1577-1585.

2. Arber DA, Orazi A, Hasserjian R, et al. The 2016 revision to the World Health Organization classification of myeloid neoplasms and acute leukemia. Blood. 2016;127:23912405.

3. Barosi G, Mesa RA, Thiele J, et al. Proposed criteria for the diagnosis of post-polycythemia vera and post-essential thrombocythemia myelofibrosis: a consensus statement from the International Working Group for Myelofibrosis Research and Treatment. Leukemia. 2008;22:437-438. 
4. Barosi G. Myelofibrosis with myeloid metaplasia: diagnostic definition and prognostic classification for clinical studies and treatment guidelines. J Clin Oncol. 1999;17:29542970.

5. Visani G, Finelli C, Castelli U, et al. Myelofibrosis with myeloid metaplasia: clinical and haematological parameters predicting survival in a series of 133 patients. Br J Haematol. 1990;75:4-9.

6. Gangat N, Caramazza D, Vaidya R, et al. DIPSS plus: a refined Dynamic International Prognostic Scoring System for primary myelofibrosis that incorporates prognostic information from karyotype, platelet count, and transfusion status. J Clin Oncol. 2011;29:392-397.

7. Passamonti F, Cervantes F, Vannucchi AM, et al. A dynamic prognostic model to predict survival in primary myelofibrosis: a study by the IWG-MRT (International Working Group for Myeloproliferative Neoplasms Research and Treatment). Blood. 2010;115:1703-1708.

8. Gowin K, Coakley M, Kosiorek H, Mesa R. Discrepancies of applying primary myelofibrosis prognostic scores for patients with post polycythemia vera/essential thrombocytosis myelofibrosis. Haematologica. 2016;101:e405-e406.

9. Mesa RA, Niblack J, Wadleigh M, et al. The burden of fatigue and quality of life in myeloproliferative disorders (MPDs): an international Internet-based survey of 1179 MPD patients. Cancer. 2007;109:68-76.

10. Tefferi A, Guglielmelli P, Larson DR, et al. Long-term survival and blast transformation in molecularly annotated essential thrombocythemia, polycythemia vera, and myelofibrosis. Blood. 2014;124:2507-2513; quiz 2615.

11. Cervantes F, Dupriez B, Passamonti F, et al. Improving survival trends in primary myelofibrosis: an international study. J Clin Oncol. 2012;30:2981-2987.

12. Helbig G, Wieczorkiewicz A, Woźniczka K, WiśniewskaPiąty K, Rusek A, Kyrcz-Krzemień S. The JAK2V617F tyrosine kinase mutation has no impact on overall survival and the risk of leukemic transformation in myelofibrosis. Med Oncol. 2012;29:2379-2384.

13. Tefferi A, Lasho TL, Huang J, et al. Low JAK2V617F allele burden in primary myelofibrosis, compared to either a higher allele burden or unmutated status, is associated with inferior overall and leukemia-free survival. Leukemia. 2008;22:756-761.

14. Tefferi A, Lasho TL, Finke CM, et al. Targeted deep sequencing in primary myelofibrosis. Blood Adv. 2016;1:105-111.

15. Rumi E, Pietra D, Pascutto $C$, et al. Clinical effect of driver mutations of JAK2, CALR, or MPL in primary myelofibrosis. Blood. 2014;124:1062-1069.

16. Tefferi A, Lasho $T$, Finke $C$, et al. CALR vs JAK2 vs MPL-mutated or triple-negative myelofibrosis: clinical, cytogenetic and molecular comparisons. Leukemia. 2014;28:1472-1477.

17. Mesa RA, Li CY, Ketterling RP, Schroeder GS, Knudson RA, Tefferi A. Leukemic transformation in myelofibrosis with myeloid metaplasia: a single-institution experience with 91 cases. Blood. 2005;105:973-977.

18. Tam CS, Nussenzveig RM, Popat $U$, et al. The natural history and treatment outcome of blast phase BCR-ABLmyeloproliferative neoplasms. Blood. 2008;112:16281637.

19. Abdulkarim K, Girodon F, Johansson P, et al. AML transformation in 56 patients with Ph- MPD in two well defined populations. Eur J Haematol. 2009;82:106-111.

20. Ballen KK, Shrestha S, Sobocinski KA, et al. Outcome of transplantation for myelofibrosis. Biol Blood Marrow Transplant. 2010;16:358-367.

21. Harrison CN, Vannucchi AM, Kiladjian JJ, et al. Long-term findings from COMFORT-II, a phase 3 study of ruxolitinib vs best available therapy for myelofibrosis. Leukemia. 2016;30:1701-1707. 